utilized to image protein activity noninvasively in animals and humans. We hypothesized that specific inflammatory processes observed in nephritis can be noninvasively detected and monitored using NIRF-based optical imaging approaches. Using a probe that becomes fluorescent in the presence of the protease cathepsin B (CatB), we tested the ability of using NIR optical imaging to assess renal inflammation as a noninvasive marker for early-stage glomerulonephritis (GN).

Methods Experimental GN was induced in 129 mice by nephrotoxic serum (NTS) delivered intravenously. Proteinuria was quantified using albumin ELISA and chromogenic creatinine assay. NIR optical imaging of anesthetized mice was performed following intravenous administration of a cleavable sensor for $\mathrm{CatB}$ and fluorescence intensity of kidney regions quantified using fluorescence molecular tomography (FMT)-3000 instrument at days 1 to 10 post-NTS administration.

Results In NTS-treated mice, a strong signal from the CatBactivatable probe was observed as early as day 1, which associated with the onset of proteinuria (figure 1). This signal could be detected for at least 10 days. In contrast, control mice were devoid of any CatB signal. To assess the specificity of CatB signal to $\mathrm{GN}$, we examined $\mathrm{CD} 2 \mathrm{AP} \mathrm{KO}$ mice that develop nephrotic syndrome in the absence of inflammation. CD2AP KO had no CatB signal despite ongoing nephrosis.

Conclusions Induction of GN by NTS was specifically detected noninvasively using a CatB-activatable probe and NIR optical imaging. These data establish the proof-of-principle that novel noninvasive tomographic approaches may represent a translatable approach to establishing early stages of GN. We believe that this approach can be expanded to other experimental imaging approaches, such as photoacoustics, as a novel method for detecting lupus nephritis in humans.

Funding Source(s): Department of Defense Discovery Award \#W81XWH-17-1-0128 (PI: Kim)

Representative images of a control mouse and a mouse treated with nephrotoxic serum to induce glomerulonephritis (NTS). A strong fluorescence signal induced by cathepsin B is observed in NTS-treated mice only.

\section{ACTIVATED STRESS RESPONSE GENES AND PERTURBATION OF REGULATORY PATHWAYS IN ANTI- NUCLEAR ANTIBODY POSITIVE INDIVIDUALS AND SLE PATIENTS VARY BY CELL TYPE AND RACE IN SINGLE- CELL TRANSCRIPTOMIC ANALYSES}

Miles Smith*, Samantha Slight-Webb, Susan Macwana, Eliza Chakravarty, Cristina Arriens, Joan T Merrill, Judith A James, Joel Guthridge. Oklahoma Medical Research Foundation

10.1136/lupus-2019-Ism.296

Background A universal hallmark of systemic lupus erythematosus (SLE) is the presence of antinuclear antibodies (ANAs). While their cognate antigens and ANA levels can associate with SLE subphenotypes, many individuals develop ANAs years prior to autoimmune disease manifestations or never develop any clinical symptoms. At present, the mechanisms governing the transition from autoantibody production to disease onset remain unknown.

Methods To ascertain differences in immune cell populations and their transcriptional state, we performed scRNA-seq on peripheral blood mononuclear cells from 30 patients, including African and European American individuals with lupus-associated autoantibodies $(n=10)$, SLE patients $(n=10)$, and ANA- healthy controls $(n=10)$. Transcriptomes were analyzed using canonical correlation analysis, the results of which were used by a smart local moving algorithm to identify cell population clusters and by Uniform Manifold Approximation and Projection for visualization. Differential expression of genes was assessed using Model-based Analysis of Single Cell Transcriptomics.

Results Distinct cell populations could be identified for each disease classification. EA ANA + individuals showed a decrease in the number of naïve $B$ cells relative to SLE and healthy individuals; in contrast, AA ANA +individuals had higher numbers of centrocytes. Additionally, AA SLE patients demonstrated an enrichment of plasma cells relative to both ANA + and healthy individuals while FCRL5, a marker of dysfunctional B cells, was increased only in SLE memory B cells. Overall, AA ANA + cell populations showed an upregulation in a number of heat shock genes in ANA+ (HSPA6, HSPA1A, DNAJB1). Multipotent progenitor (MPP) cells from SLE patients of both ethnicities were marked by upregulation of interferon stimulated genes; ANA +MPPs, however, differed in that cells from AA individuals had an upregulation of housekeeping genes (GAPDH, PGK1) while anti-inflammatory NFKB1A was increased in EA MPPs. Comparison of a manually curated list of regulatory factors demonstrated dysregulation of several genes, including upregulation of TGFB across all AA ANA + cell clusters and an increase in CD46 in EA ANA + clusters. Similarly, gene set enrichment analysis of differentially regulated genes indicated activation of stress response pathways in AA and EA ANA +individuals.

Conclusions These data indicate that substantial transcriptional and cell population differences exist among immune cells from ANA+, SLE, and healthy individuals, including an activated stress response and enhanced immune regulation in the cells from ANA +individuals. Our results suggest that cells from ANA +individuals may be regulating reactions to stimulation, and exhaustion of that response may allow for transition to SLE.

Funding Source(s): NIH U54GM104938, U01AI101934, U19AI082714, and P30AR053483.

\section{ENVIRONMENTAL AND ATMOSPHERIC FACTORS IN SYSTEMIC LUPUS ERYTHEMATOSUS: A REGRESSION ANALYSIS}

${ }^{1}$ George Stojan*, ${ }^{2}$ Anton Kvit, ${ }^{2}$ Frank Curriero, ${ }^{3}$ Michelle Petri. ${ }^{1}$ Johns Hopkins University; ${ }^{2}$ Bloomberg School of Public Health; ${ }^{3}$ Johns Hopkins University School of Medicine

10.1136/lupus-2019-Ism.297

Background Understanding the role of environmental exposures in the development of SLE and their association with SLE activity may help identify modifiable risk factors and potential etiological mechanisms. We hypothesized that changes in fine particulate matter (PM2.5) concentration, ozone concentration, temperature, resultant wind, relative humidity, and barometric pressure are predictive of organ specific flares in lupus.

Methods 1628 patients who fulfill 4 of the 11 ACR or SLICC classification criteria for SLE were included in the analysis. The data ranged from 1999 to 2017. Maximum distance between visits was 110 days with 1 month time aggregation units. Disease activity was expressed as Physician Global Estimate (PGA), taken at every patient visit. A flare was defined as a PGA score increase of 1 point or more compared to the previous visit. 
Environmental and atmospheric data was obtained from the EPA, including PM2.5 and ozone concentration, temperature, residual wind, relative humidity, and barometric pressure. The average values of each factor 10 days prior to patient visit was calculated. Univariate and multivariate models were built in order to study the association of these variables with lupus disease activity. The models were adjusted for age, sex, income, racial distribution, and rural vs. urban patient residence. Multivariate logistic regression was used to identify significant determinants associated with lupus flares. Regression was performed for each organ flare outcome. Regression inference was based on generalized estimating equations (GEE) to account for the time repeated outcomes.

Results Rash, serositis, hematologic, and joint flares were statistically significantly associated $(\mathrm{p}<0.00 .5)$ with an increase in temperature in univariate and multivariate analysis. Renal flares were negatively associated with increases in temperature $(\mathrm{p}<0.05)$ in univariate and multivariate analysis.

PM2.5 concentration was significantly associated $(p<0.001)$ with rash, joints, serositis, neurologic, pulmonary, and hematologic flares in univariate and multivariate analysis.

Ozone concentration, residual wind, and relative humidity were significantly associated with lupus flares in univariate analysis only, while barometric pressure had no associations.

Conclusions There is a strong association between changes in PM2.5 concentration and temperature 10 days prior to patient visit and organ specific lupus activity at the visit. These data could add an important aspect to lupus trials, the outcomes of which may be affected by so far unrecognized environmental factors, and ultimately it could allow predictive modelling of lupus flares which would revolutionize the approach to treatment.

Funding Source(s): The Hopkins Lupus Cohort was funded by AR 69572

\section{CARDIOVASCULAR RISK FACTORS IN A SYSTEMIC LUPUS ERYTHEMATOSUS COHORT FORM COLOMBIA}

${ }^{1}$ Sebastian Herrera*, ${ }^{2}$ Juan C Díaz-Coronado, ${ }^{3}$ Deicy Hernandez Parra, ${ }^{4}$ Laura BetancurVásquez, ${ }^{4}$ Jorge Lacouture-fierro, ${ }^{4}$ Daniel Gonzalez-Hurtado, ${ }^{4}$ Laura Uribe-Arango, ${ }^{4}$ Maria Fernanda Saavedra-Chacón, ${ }^{4}$ Santiago Monsalve-Yepes, ${ }^{4}$ Sebastián Guerra-Zarama, ${ }^{4}$ José David Serna-Giraldo, ${ }^{4} J u a n$ David López-López, ${ }^{4} J u l i a ́ n$ Barbosa-Arana, ${ }^{2}$ Ricardo A PinedaTamayo. 'Grupo información clínica, ARTMEDICA; ${ }^{2}$ Grupo de Información Clínica Artmedica IPS; ${ }^{3}$ ARTMEDICA; ${ }^{4}$ Departamento medicina interna, Universidad CES

\subsection{6/lupus-2019-|sm.298}

Background Systemic lupus erythematosus (SLE) is a chronic and multisystemic autoimmune disease. Higher prevalence of traditional and disease associated risk factors, such as corticosteroids and accelerated atherosclerosis due to chronic inflammation, result in an increased cardiovascular risk. Age and

Abstract 298 Table 1 Bivariate model with cardiovascular risk as dependent variable

\begin{tabular}{|c|c|c|c|}
\hline Variable & Crude OR & IC $95 \%$ & $P$ value \\
\hline \multicolumn{4}{|l|}{ Age in years } \\
\hline $36-45$ & 1,7 & $1,13-2,64$ & 0,001 \\
\hline $46-55$ & 3,5 & $2,41-5,06$ & 0,001 \\
\hline $56-65$ & 8,2 & $5,44-12,55$ & 0,001 \\
\hline$>66$ & 12,3 & $7,00-21,82$ & 0,001 \\
\hline \multicolumn{4}{|l|}{ Civil status } \\
\hline Married & 0,9 & $0,74-1,22$ & 0,733 \\
\hline Single & 1,2 & $1,05-1,59$ & 0,01 \\
\hline \multicolumn{4}{|l|}{ Education years } \\
\hline $0-5$ & 1 & & \\
\hline $6-11$ & 2,9 & $2,07-4,22$ & 0,001 \\
\hline$>12$ & 1,6 & $1,23-2,25$ & 0,001 \\
\hline Positive ds-DNA & 0,7 & $0,57-0,96$ & 0,025 \\
\hline Hypocomplementemia & 0,6 & $0,51-0,85$ & 0,001 \\
\hline \multicolumn{4}{|c|}{ Antimalarial use } \\
\hline Chloroquine & 0,81 & $0,62-1,05$ & 0,11 \\
\hline Hydroxychloroquine & 0,85 & $0,62-1,16$ & 0,31 \\
\hline \multicolumn{4}{|l|}{ Years of antimalarial use } \\
\hline $1-5$ & 0,91 & $0,64-1,29$ & 0,61 \\
\hline $6-10$ & 0,47 & $0,34-0,64$ & 0,001 \\
\hline$>11$ & 0,44 & $0,31-0,63$ & 0,001 \\
\hline \multicolumn{4}{|c|}{ Other medication } \\
\hline Corticosteroids & 2,2 & $1,32-3,80$ & 0,02 \\
\hline Cyclophosphamide & 0,5 & $0,24-1,33$ & 0,19 \\
\hline Mycophenolate & 1,1 & $0,64-2,06$ & 0,62 \\
\hline Rituximab & 1,3 & $0,49-3,47$ & 0,58 \\
\hline
\end{tabular}

